\title{
New Correlation for Calculating Critical Pressure of Petroleum Fractions
}

\author{
Sayed Gomaa, $\mathbf{P h D}^{1,2}$ \\ Mining and Petroleum Engineering Department, Faculty of Engineering, Al-Azhar University, Cairo, Egypt ${ }^{1}$ \\ Petroleum Engineering and Gas Technology Department, Faculty of Engineering, British University in Egypt ${ }^{2}$
}

\begin{abstract}
Critical pressure is one of the most important physical properties of the petroleum fractions, which commonly considered in compositional modeling studies and phase behavior calculations. This paper presents a comparison study among ten different correlations used to calculate the critical pressure of undefined petroleum fractions. A new correlation was developed for calculating the critical pressure of petroleum fractions as a function of the number of carbon atoms with an average error of $0.933977 \%$ and correlation coefficient of 0.999585 .
\end{abstract}

Keywords: Critical pressure, petroleum fractions, Compositional Modeling Studies, Phase Behavior Calculations.

\section{INTRODUCTION}

Katz and Firoozabadi [1] presented a generalized set of physical properties for the petroleum fractions $\mathrm{C} 6$ through C45. The tabulated properties include the critical molecular weight. The authors generated these properties by analyzing the physical properties of 26 condensates and crude oil samples. These generalized properties are given in Table A-1.

Ahmed [2, 3] correlated Katz-Firoozabadi-tabulated physical properties with the number of carbon atoms of the fraction by using a regression model. The generalized equation has the following form:

$$
P_{c}=a_{1}+a_{2} n+a_{3} n^{2}+a_{4} n^{3}+a_{5} / n
$$

Where:

$$
\begin{array}{ll}
\mathrm{a}_{1}=311.2361908 & \mathrm{a}_{2}=-14.6869301 \\
\mathrm{a}_{3}=0.3287671 & \\
\mathrm{a}_{4}=-0.0027346 & \mathrm{a}_{5}=1690.9001135
\end{array}
$$

\section{Undefined Petroleum Fractions}

Nearly all naturally occurring hydrocarbon systems contain a quantity of heavy fractions that are not well defined and are not mixtures of discretely identified components. These heavy fractions are often lumped together and identified as the plus fraction, e.g., $\mathrm{C}_{7}$ fraction $[2,4]$.

A proper description of the physical properties of the plus fractions and other undefined petroleum fractions in hydrocarbon mixtures is essential in performing reliable phase behavior calculations and compositional modeling studies. Frequently, a distillation analysis or a chromatographic analysis is available for this undefined fraction. Other physical properties, such as molecular weight and specific gravity, may also be measured for the entire fraction or for various cuts of it $[3,5]$.

To use any of the thermodynamic property-prediction models, e.g., equations, of state, to predict the phase and volumetric behavior of complex hydrocarbon mixtures, one must be able to provide the acentric factor, along with the critical temperature and critical pressure, for both the defined and undefined (heavy) fractions in the mixture. The problem of how to adequately characterize these undefined plus fractions in terms of their critical properties and acentric factors has been long recognized in the petroleum industry $[3,5]$.

Riazi and Daubert [6] developed a simple two-parameter equation for predicting the physical properties of pure compounds and undefined hydrocarbon mixtures. The proposed generalized empirical equation is based on the use of the molecular weight and specific gravity of the undefined petroleum fraction as the correlating parameters. Their mathematical expression has the following form:

$$
\mathrm{P}_{\mathrm{c}}=\mathrm{aM}^{\mathrm{b}} \gamma^{\mathrm{c}} \operatorname{EXP}(\mathrm{dM}+\mathrm{e} \gamma)
$$

Where:

$$
\begin{array}{lll}
a=45203 & b=-0.8063 & c= \\
1.6015 & & \\
d=-0.0018078 & e=-0.3084 &
\end{array}
$$

Edmister [7] proposed a correlation for estimating the acentric factor of pure fluids and petroleum fractions. The equation, widely used in the petroleum industry, requires boiling point, critical temperature, and critical pressure. The proposed expression is given by the following relationship:

$$
\omega=\frac{3\left[\log \left(\mathrm{p}_{\mathrm{c}} / 14.7\right)\right]}{7\left[\left(\mathrm{~T}_{\mathrm{c}} / \mathrm{T}_{\mathrm{b}}\right)-1\right]}-1
$$

The Edmister equation can be rearranged to solve for the critical pressure as follows:

$$
\begin{aligned}
\log \left(\mathrm{p}_{\mathrm{c}}\right) & =\log (14.7)+\left(\frac{7}{3}\right) *(\omega+1) \\
* & {\left.\left[\left(\mathrm{~T}_{\mathrm{c}} / \mathrm{T}_{\mathrm{b}}\right)-1\right)\right] }
\end{aligned}
$$

Cavett [8] proposed correlations for estimating the critical pressure of hydrocarbon fractions. The correlations 
Vol. 3, Issue 11, November 2016

received wide acceptance in the petroleum industry due to their reliability in extrapolating conditions beyond those of the data used in developing the correlations. The proposed correlations were expressed analytically as functions of the normal boiling point in ${ }^{\circ} \mathrm{F}$ and API gravity. Cavett proposed the following expression for estimating the critical pressure of petroleum fractions:

$$
\begin{aligned}
\log \left(\mathrm{p}_{\mathrm{c}}\right)=\mathrm{b}_{0} & +\mathrm{b}_{1}\left(\mathrm{~T}_{\mathrm{b}}\right)+\mathrm{b}_{2}\left(\mathrm{~T}_{\mathrm{b}}\right)^{2}+\mathrm{b}_{3}(\mathrm{API})\left(\mathrm{T}_{\mathrm{b}}\right) \\
& +\mathrm{b}_{4}\left(\mathrm{~T}_{\mathrm{b}}\right)^{3}+\mathrm{b}_{5}(\mathrm{API})\left(\mathrm{T}_{\mathrm{b}}\right)^{2} \\
& \left.+\mathrm{b}_{6}(\mathrm{API})^{2}\left(\mathrm{~T}_{\mathrm{b}}\right)+\mathrm{b}_{7}(\mathrm{API})^{2}\left(\mathrm{~T}_{\mathrm{b}}\right)^{2} 5\right)
\end{aligned}
$$

Kesler and Lee [9] proposed a correlation to estimate the critical pressure of petroleum fractions. This relationship use specific gravity boiling point as input parameters for their proposed expressions:

$$
\begin{aligned}
& \ln \left(p_{c}\right)=8.3634-0.0566 / \gamma-\left(0.24244+2.2898 / \gamma+0.11857 / \gamma^{2}\right) \times 10^{-3} T_{b}+(1.4685 \\
& \left.+3.648 / \gamma+0.47227 / \gamma^{2}\right) \times 10^{-7} T_{b}{ }^{2}-\left(0.42019+1.6977 / \gamma^{2}\right) \times 10^{-10} T_{b}{ }^{3}(6)
\end{aligned}
$$

Winn [10] developed convenient nomographs to estimate Proposed correlation

various physical properties including molecular weight A new correlation was developed by use of the linear and and the pseudocritical pressure for petroleum fractions. Sim and Daubert [11] developed analytical relationships that closely matched the monograph graphical data. The authors used specific gravity and boiling point as the correlating parameters for calculating the critical pressure of the undefined petroleum fraction:

$$
p_{c}=3.48242 \times 10^{9} T_{b}^{-2.3177} \gamma^{2.4853}
$$

Watansiri et al. [12] developed a set of correlations to estimate the critical properties and acentric factor of coal compounds and other undefined hydrocarbon components and their derivatives. The proposed correlations express the critical and physical properties of the undefined fraction as a function of the fraction normal boiling point, specific gravity, and molecular weight. These relationships have the following forms:

$$
\begin{aligned}
& \ln \left(p_{c}\right)=6.6418853+0.01617283\left(T_{c} / V_{c}\right)^{0.8} \\
& -8.712\left(M / T_{c}\right) 0.08843889\left(T_{b} / M\right)(8)
\end{aligned}
$$

Willman and Teja [13] proposed correlation for determining the critical pressure of the n-alkane homologous series:

$$
\begin{gathered}
p_{c}=(339.0416805+1184.157759 n)[0.87359 \\
+0.54285 n]^{-1.9265669}
\end{gathered}
$$

Lin and Chao [14] developed a correlation to estimate the critical pressure as a function of molecular weight:

$$
p_{c}=C_{1}+C_{2} M+C_{3}(M)^{2}+C_{4}(M)^{3}+C_{5} / M
$$

Where:

$$
\begin{aligned}
& C_{1}=6.753444 \quad C_{2}=-0.010182 \quad C_{3}= \\
& 0.0000251106 \\
& C_{4}=-0.0000000373776 \quad C_{5}=3.50737
\end{aligned}
$$

Sancet [15] presented a correlation to estimate the critical pressure from the molecular weight. This correlation has the following form:

$$
p_{c}=653 \exp (-0.007427 M)+82.82
$$

nonlinear regression analysis and can be expressed as:

$$
\begin{gathered}
P_{c}=a_{0}+a_{1} \ln (n)+a_{2}[\ln (n)]^{2}+a_{3}[\ln (n)]^{3}+a_{4} n \\
+a_{5} n^{2}+a_{6} n^{3}+a_{7} / n
\end{gathered}
$$

With the coefficients $a_{0}$ through $a_{7}$ having the following values:

$a_{0}=-50662.795181 \quad a_{1}=50832.035066$

$a_{2}=-17678.300681$

$a_{3}=3393.832113 \quad a_{4}=-1991.986187 \quad a_{5}=$

$8.395313 a_{6}=-0.02554 \quad a_{7}=53728.166034$

\section{Statistical Error analysis}

The statistical error analyses were used to check the accuracy of the critical pressure correlations developed by Ahmed, Reazi- Daubert, Lin, Cavett, Kessler-Lee, WinnSim, Watansiri, Willman, Sancet and this study.

The accuracy of correlations relative to the experimental values tabulated by Katz-Firoozabadi-tabulated is determined by various statistical means. The criteria used in this study were average percent relative error, average absolute percent relative error, minimum/maximum absolute percent relative error, standard deviation, and the correlation coefficient.

\section{Average Relative Error}

This is an indication of the relative deviation in percent from the experimental values and is given by:

$$
\left(\sum_{i=1}^{n} E_{i}\right) / n
$$

$E_{i}$ is the relative deviation in percent of an estimated value from an experimental value and is defined by:

$$
\mathrm{E}_{\mathrm{i}}=\left[\frac{\left(\mathrm{p}_{\mathrm{c}_{\exp }}-\mathrm{p}_{\mathrm{c}_{\mathrm{cal}}}\right)}{\mathrm{p}_{\mathrm{c}_{\exp }}}\right]_{\mathrm{i}} \times 100
$$


Vol. 3, Issue 11, November 2016

The lower the value of $E_{i}$ the more equally distributed are the errors between positive and negative values.

\section{Average Absolute Relative Error}

This is defined as:

$$
\sum_{i=1}^{n}\left|E_{i}\right| / n
$$

and indicates the relative absolute deviation in percent from the tabulated values. A lower value implies a better correlation.

\section{Standard Deviation}

Standard deviation $S_{\mathrm{x}}$ is a measure of dispersion and is expressed as:

$$
\mathrm{s}^{2}{ }_{\mathrm{x}}=\left(\sum_{\mathrm{i}=1}^{\mathrm{n}} \mathrm{E}_{\mathrm{i}}^{2}\right) /(\mathrm{n}-1)
$$

A lower value of standard deviation means a smaller degree of scatter.

\section{Correlation Coefficient}

The correlation coefficient, r, represents the degree of success in reducing the standard deviation by regression analysis. It is defined as:

$$
\mathrm{r}^{2}=1-\left[\sum_{\mathrm{i}=1}^{\mathrm{n}}\left(\mathrm{p}_{\mathrm{c}_{\text {cal }}}-\mathrm{p}_{\mathrm{c}_{\text {exp }}}\right)^{2} / \sum_{\mathrm{i}=1}^{\mathrm{n}}\left(\mathrm{p}_{\mathrm{c}_{\mathrm{cal}}}-\mathrm{p}_{\mathrm{c}_{\mathrm{avg}}}\right)^{2}\right]
$$

Where

$$
\left.\mathrm{p}_{\mathrm{c}_{\text {avg }}}=\left(\sum_{\mathrm{i}=1}^{\mathrm{n}} \mathrm{p}_{\mathrm{ci}}\right)_{\exp }\right) / \mathrm{n}
$$

The correlation coefficient lies between 0 and 1 . A value of 1 indicates a perfect correlation, whereas a value of 0 implies no correlation at all among the given independent variables.

\section{Comparison of Correlations}

Statistical Error Analysis

Average relative error, average absolute relative error, standard deviation, and correlation coefficient were computed for each correlation.

Table 1 presents the comparison of errors relative to the experimental critical pressure calculated from two correlations. The correlation for critical pressure of this

\begin{tabular}{|c|c|c|c|c|c|}
\hline Experimental & Reazi & Lin & Cavett & Ahmed & Sayed \\
\hline 483 & 486.5995 & 443.6061 & 474.3079714 & 516.1762 & 483.2575 \\
\hline 453 & 459.5854 & 407.8327 & 454.7675179 & 465.1565 & 452.7127 \\
\hline 419 & 430.0627 & 379.347 & 431.8667081 & 424.7442 & 417.6729 \\
\hline 383 & 392.9715 & 347.8725 & 405.8220313 & 391.5682 & 383.6256 \\
\hline 351 & 362.3341 & 322.5254 & 378.6496158 & 363.599 & 352.8333 \\
\hline 325 & 334.7507 & 300.1955 & 354.2682187 & 339.5392 & 325.9901 \\
\hline 302 & 309.017 & 278.8939 & 331.0501626 & 318.5184 & 303.0472 \\
\hline 286 & 286.9266 & 259.908 & 312.4320969 & 299.9291 & 283.6288 \\
\hline 270 & 266.1019 & 241.6387 & 293.8190942 & 283.3324 & 267.2419 \\
\hline 255 & 246.1526 & 224.0397 & 275.255954 & 268.4022 & 253.3796 \\
\hline 241 & 227.6728 & 207.9795 & 258.1837004 & 254.8900 & 241.5707 \\
\hline 230 & 212.8811 & 194.0224 & 243.6370851 & 242.6017 & 231.3995 \\
\hline 222 & 199.7345 & 181.7564 & 232.2516263 & 231.3827 & 222.5123 \\
\hline 214 & 189.6965 & 171.7151 & 222.0847588 & 221.1076 & 214.6148 \\
\hline 207 & 180.4563 & 162.033 & 211.3101072 & 211.6726 & 207.4679 \\
\hline 200 & 168.788 & 149.5843 & 201.7427904 & 202.9909 & 200.8809 \\
\hline 193 & 163.2755 & 142.7784 & 193.2726173 & 194.9881 & 194.7048 \\
\hline 188 & 155.9749 & 133.8956 & 185.7979324 & 187.6001 & 188.8262 \\
\hline 182 & 148.9473 & 125.213 & 178.6940495 & 180.7708 & 183.1615 \\
\hline 177 & 141.5802 & 116.0197 & 171.5284832 & 174.4503 & 177.6515 \\
\hline 173 & 135.4937 & 107.7274 & 165.7204516 & 168.5939 & 172.2577 \\
\hline 169 & 130.3144 & 100.2945 & 160.6576099 & 163.1611 & 166.9576 \\
\hline 165 & 124.7418 & 92.38068 & 155.4741815 & 158.1149 & 161.7424 \\
\hline 161 & 120.4446 & 85.95315 & 151.634223 & 153.4211 & 156.6132 \\
\hline 158 & 115.4629 & 78.45942 & 147.3897367 & 149.0478 & 151.5795 \\
\hline 143 & 111.7754 & 72.41387 & 144.1224668 & 144.9652 & 146.6569 \\
\hline 138 & 107.6944 & 65.99212 & 140.7006346 & 141.1452 & 141.8655 \\
\hline 134 & 103.8193 & 59.82957 & 137.7846727 & 137.5609 & 137.2282 \\
\hline
\end{tabular}
study achieved the highest correlation coefficient accuracy of 0.999585 , as presented in Table 2 .

Table 1 Comparison of critical pressure calculated by correlations from this study and others 
International Advanced Research Journal in Science, Engineering and Technology ISO 3297:2007 Certified

Vol. 3, Issue 11, November 2016

\begin{tabular}{|r|r|r|r|r|r|}
\hline 130 & 99.99206 & 53.94648 & 134.9703598 & 134.1870 & 132.7701 \\
\hline 127 & 97.54432 & 49.85437 & 132.9233977 & 130.9988 & 128.5172 \\
\hline 124 & 94.02806 & 44.49788 & 130.474535 & 127.9728 & 124.4954 \\
\hline 121 & 91.77916 & 40.8066 & 128.9424617 & 125.0862 & 120.7303 \\
\hline 118 & 88.53959 & 36.02199 & 127.0247512 & 122.3169 & 117.2463 \\
\hline 115 & 86.04582 & 32.36387 & 125.4438954 & 119.6434 & 114.0663 \\
\hline 112 & 83.07149 & 28.2116 & 123.8322733 & 117.0444 & 111.2112 \\
\hline 110 & 81.3324 & 25.75291 & 122.8347137 & 114.4997 & 108.6998 \\
\hline 108 & 78.72571 & 22.48766 & 121.4980115 & 111.9888 & 106.5483 \\
\hline 105 & 76.58938 & 19.79505 & 120.5610443 & 109.4920 & 104.7704 \\
\hline 103 & 74.28081 & 17.07123 & 119.4965043 & 106.9898 & 103.377 \\
\hline 101 & 72.53922 & 15.08983 & 118.908402 & 104.4628 & 102.3759 \\
\hline
\end{tabular}

Table 1 Cont.: Comparison of critical pressure calculated by correlations from this study and others

\begin{tabular}{|c|c|c|c|c|c|}
\hline Kessler & Winn-Simm & Edmister & Watansiri & Willman & Sancet \\
\hline 475.7941 & 490.6552 & 484.968823 & 494.0948601 & 484.1689 & 432.7416 \\
\hline 455.7638 & 463.3941 & 448.3040413 & 463.6339024 & 442.3843 & 402.9045 \\
\hline 427.4347 & 429.512 & 420.5805151 & 434.4403965 & 407.108 & 377.7943 \\
\hline 396.4306 & 394.5733 & 384.0602243 & 396.2830547 & 377.015 & 348.6641 \\
\hline 366.3598 & 362.547 & 350.0630266 & 364.8704104 & 351.0822 & 324.1968 \\
\hline 341.0123 & 336.6751 & 326.0504211 & 336.9122923 & 328.5233 & 301.9813 \\
\hline 318.1025 & 314.0271 & 303.4746434 & 310.1766626 & 308.7307 & 280.3381 \\
\hline 300.6279 & 297.1088 & 284.9497729 & 286.4527956 & 291.2303 & 260.8322 \\
\hline 283.8007 & 281.2067 & 268.887675 & 264.1577419 & 275.6485 & 242.0655 \\
\hline 267.3945 & 266.1107 & 255.6445822 & 242.9863424 & 261.6873 & 224.2232 \\
\hline 252.27 & 252.5976 & 241.5604987 & 222.8809163 & 249.1068 & 208.3799 \\
\hline 239.7186 & 241.507 & 230.9518626 & 206.9204758 & 237.7118 & 195.1429 \\
\hline 229.7009 & 232.8445 & 221.1620973 & 192.1894556 & 227.3419 & 184.0505 \\
\hline 220.7771 & 225.2051 & 213.9721549 & 181.3967283 & 217.8645 & 175.4188 \\
\hline 211.1687 & 217.092 & 205.7912628 & 171.65114 & 209.1686 & 167.5231 \\
\hline 202.5909 & 209.9168 & 199.8857102 & 158.7385351 & 201.1609 & 158.0327 \\
\hline 194.9432 & 203.5703 & 192.8457201 & 153.3087592 & 193.7624 & 153.1696 \\
\hline 188.1387 & 197.961 & 187.8318369 & 145.5723076 & 186.9058 & 147.171 \\
\hline 181.3904 & 192.4708 & 181.9426756 & 138.0179495 & 180.5331 & 141.684 \\
\hline 174.2314 & 186.7235 & 177.9829364 & 130.413171 & 174.5947 & 136.2663 \\
\hline 168.4868 & 182.1078 & 172.870038 & 123.8364352 & 169.0471 & 131.7091 \\
\hline 163.3878 & 178.0275 & 169.4158311 & 118.4605784 & 163.8528 & 127.8738 \\
\hline 157.8294 & 173.634 & 165.1475023 & 112.5653569 & 158.9788 & 124.0322 \\
\hline 153.6697 & 170.3481 & 160.8962772 & 107.9064729 & 154.3961 & 121.0822 \\
\hline 148.8598 & 166.575 & 158.4226723 & 102.7690901 & 150.0791 & 117.8197 \\
\hline 145.1926 & 163.6818 & 143.6578274 & 98.98044296 & 146.0052 & 115.3144 \\
\hline 141.0457 & 160.4491 & 138.5137221 & 94.69497994 & 142.1542 & 112.7653 \\
\hline 137.3853 & 157.6011 & 134.8857603 & 90.68844035 & 138.5081 & 110.4161 \\
\hline 133.6109 & 154.6899 & 130.4651316 & 86.63458303 & 135.0509 & 108.2512 \\
\hline 130.8394 & 152.5374 & 127.2683332 & 84.2220399 & 131.7681 & 106.7842 \\
\hline 127.2556 & 149.7861 & 123.3277198 & 80.55322596 & 128.6467 & 104.9043 \\
\hline 124.9567 & 148.0047 & 121.4809425 & 78.399966 & 125.6749 & 103.6303 \\
\hline 121.8599 & 145.6341 & 117.8412754 & 74.96735094 & 122.8422 & 101.9978 \\
\hline 119.152 & 143.5625 & 115.2558872 & 72.50478695 & 120.139 & 100.7578 \\
\hline 116.2044 & 141.3132 & 111.9798104 & 69.40769228 & 117.5564 & 99.35058 \\
\hline 114.221 & 139.7958 & 110.5923539 & 67.8061103 & 115.0864 & 98.51313 \\
\hline 111.4857 & 137.7213 & 107.5755948 & 65.1006677 & 112.7219 & 97.38983 \\
\hline 109.3013 & 136.0562 & 105.442655 & 62.97507318 & 110.456 & 96.44778 \\
\hline 106.6034 & 134.0058 & 102.5297079 & 60.70537044 & 108.2828 & 95.47231 \\
\hline 104.793 & 132.6266 & 100.6609292 & 58.97903267 & 106.1965 & 94.74246 \\
\hline
\end{tabular}


Vol. 3, Issue 11, November 2016

Table 2 Statistical accuracy of critical pressure correlations

\begin{tabular}{|l|c|c|c|}
\hline & AARE, \% & SD & $\mathrm{R}^{2}$ \\
\hline This study(sh4) & 0.933977 & 1.317814 & 0.999585 \\
\hline Ahmed & 3.475806 & 3.838117 & 0.991964 \\
\hline Reazi & 16.22215 & 19.16977 & 0.946263 \\
\hline Lin & 39.34908 & 47.73011 & 0.773554 \\
\hline Cavett & 6.191865 & 7.612793 & 0.981904 \\
\hline Kesler & 3.169919 & 3.518671 & 0.99421 \\
\hline Winn-Simm & 11.5529 & 15.28577 & 0.962153 \\
\hline Edmister & 0.322695 & 0.387337 & 0.999887 \\
\hline Watansiri & 22.71815 & 27.04131769 & 0.910100284 \\
\hline Willman & 2.809648 & 0.032314 & 0.996947 \\
\hline Sancet & 15.12048 & 16.41881 & 0.896163 \\
\hline
\end{tabular}

\section{CONCLUSIONS}

From this paper, one may conclude that:

1. This paper presents a comparison among nine different correlations used to calculate the critical pressure of undefined petroleum fractions.

2. New correlation was developed for calculating the critical pressure of undefined petroleum fractions.

3. Deviations from experimental values of critical pressure indicated as average percent relative errors, average absolute percent relative errors, and the standard deviations, were lower for this study than for calculated values based on Ahmed, Reazi- Daubert, Lin, Cavett, Kessler-Lee, Winn-Sim, Watansiri and Willman, Sancet correlations.

3. The developed correlation is more practical than Edmister correlation, which is a function of critical temperature and acentric factor that in turn need two correlations to be calculated.

4. The correlation coefficient of the correlations of this study are closer to one than that of other correlations.

\section{Nomenclature}

$\mathrm{p}_{\mathrm{c}}=$ critical pressure, psia

$\mathrm{T}_{\mathrm{c}}=$ critical temperature, ${ }^{\circ} \mathrm{R}$

$\mathrm{T}_{\mathrm{b}}=$ boiling point, ${ }^{\circ} \mathrm{R}$

$\omega=$ acentric factor

$\mathrm{M}=$ molecular weight

$\gamma=$ specific gravity

$\mathrm{v}_{\mathrm{c}}=$ critical volume, $\mathrm{ft}^{3} / \mathrm{lb}-\mathrm{mol}$

$\mathrm{n}=$ no of carbon atoms

\section{REFERENCES}

1. Katz, D.L., Firoozabadi, A., 1978. Predicting phase behavior of condensate/crude-oil systems using methane interaction coefficients. J. Petrol. Tech. (November), 1649-1655.

2. Ahmed, T., Hydrocarbon Phase Behavior, Gulf Publishing Company, 1989.

3. Ahmed, T., Equations of State and PVT Analysis Applications for Improved Reservoir Modeling, Amsterdam: Elsevier, second edition 2016.

4. Danesh, A., PVT and Phase Behavior of Petroleum Reservoir Fluids, Elsevier Science \& Technology Books, 1998.

5. Whitson, C. H. and Brule, M. R., Phase Behavior, SPE, Texas, 2000

6. Riazi, M.R., Daubert, T.E., 1987. Characterization parameters for petroleum fractions. Ind. Eng. Chem. Fundam. 26 (24), 755-759.

Edmister, W.C., 1958. Applied hydrocarbon thermodynamics, part 4, compressibility factors and equations of state. Petroleum Refiner. 37 (April), 173-179.

8. Cavett, R.H., 1962. Physical data for distillation calculationsvapor-liquid equilibrium, Proceedings of the 27th Meeting, API, San Francisco, pp. 351-366

9. Kesler, M.G., Lee, B.I., 1976. Improve prediction of enthalpy of fractions. Hydrocarb. Process. (March), 153-158.

10. Winn, F.W., 1957. Simplified nomographic presentation, characterization of petroleum fractions. Petroleum Refiner. 36 (2), 157.

11. Sim, W.J., Daubert, T.E., 1980. Prediction of vapor-liquid equilibria of undefined mixtures. Ind. Eng. Chem. Process. Des. Dev. 19 (3), 380-393

12. Watansiri, S., Owens, V.H., Starling, K.E., 1985 Correlations for estimating critical constants, acentric factor, and dipole moment for undefined coal-fluid fractions. Ind. Eng. Chem. Process. Des. Dev. 24, 294-296.

13. Willman, B., Teja, A., 1987. Prediction of dew points of semicontinuous natural gas and petroleum mixtures. Ind. Eng. Chem. Res. 226 (5), 948-952.

14. Lin, H. M. and Chao, K. C., Correlation of critical properties and acentric factor of hydrocarbons and derivatives, AIChE Journal, Vol. 30, No. 6, Vov. 1984, PP. 981-983

15. Sancet, J., Heavy Faction Characterization. In: SPE 113025, 2007 SPE Annual Conference, November 11-14, Anaheim, CA 2007.

\section{Appendix 1:}

Table A-1

\begin{tabular}{|c|c|c|c|c|c|c|c|c|}
\hline $\mathbf{C}$ & Tb & SG & K & M & Tc & Pc & w & Vc \\
\hline 6 & 607 & 0.69 & 12.27 & 84 & 923 & 483 & 0.25 & 0.06395 \\
\hline 7 & 658 & 0.727 & 11.96 & 96 & 985 & 453 & 0.28 & 0.06289 \\
\hline 8 & 702 & 0.749 & 11.87 & 107 & 1,036 & 419 & 0.312 & 0.06264 \\
\hline 9 & 748 & 0.768 & 11.82 & 121 & 1,085 & 383 & 0.348 & 0.06258 \\
\hline
\end{tabular}


International Advanced Research Journal in Science, Engineering and Technology

ISO 3297:2007 Certified

Vol. 3, Issue 11, November 2016

\begin{tabular}{|c|c|c|c|c|c|c|c|c|}
\hline 10 & 791 & 0.782 & 11.83 & 134 & 1,128 & 351 & 0.385 & 0.06273 \\
\hline 11 & 829 & 0.793 & 11.85 & 147 & 1,166 & 325 & 0.419 & 0.06291 \\
\hline 12 & 867 & 0.804 & 11.86 & 161 & 1,203 & 302 & 0.454 & 0.06306 \\
\hline 13 & 901 & 0.815 & 11.85 & 175 & 1,236 & 286 & 0.484 & 0.06311 \\
\hline 14 & 936 & 0.826 & 11.84 & 190 & 1,270 & 270 & 0.516 & 0.06316 \\
\hline 15 & 971 & 0.836 & 11.84 & 206 & 1,304 & 255 & 0.55 & 0.06325 \\
\hline 16 & 1,002 & 0.843 & 11.87 & 222 & 1,332 & 241 & 0.582 & 0.06342 \\
\hline 17 & 1,032 & 0.851 & 11.87 & 237 & 1,360 & 230 & 0.613 & 0.0635 \\
\hline 18 & 1,055 & 0.856 & 11.89 & 251 & 1,380 & 222 & 0.638 & 0.06362 \\
\hline 19 & 1,077 & 0.861 & 11.91 & 263 & 1,400 & 214 & 0.662 & 0.06372 \\
\hline 20 & 1,101 & 0.866 & 11.92 & 275 & 1,421 & 207 & 0.69 & 0.06384 \\
\hline 21 & 1,124 & 0.871 & 11.94 & 291 & 1,442 & 200 & 0.717 & 0.06394 \\
\hline 22 & 1,146 & 0.876 & 11.95 & 300 & 1,461 & 193 & 0.743 & 0.06402 \\
\hline 23 & 1,167 & 0.881 & 11.95 & 312 & 1,480 & 188 & 0.768 & 0.06408 \\
\hline 24 & 1,187 & 0.885 & 11.96 & 324 & 1,497 & 182 & 0.793 & 0.06417 \\
\hline 25 & 1,207 & 0.888 & 11.99 & 337 & 1,515 & 177 & 0.819 & 0.06431 \\
\hline 26 & 1,226 & 0.892 & 12 & 349 & 1,531 & 173 & 0.844 & 0.06438 \\
\hline 27 & 1,244 & 0.896 & 12 & 360 & 1,547 & 169 & 0.868 & 0.06443 \\
\hline 28 & 1,262 & 0.899 & 12.02 & 372 & 1,562 & 165 & 0.894 & 0.06454 \\
\hline 29 & 1,277 & 0.902 & 12.03 & 382 & 1,574 & 161 & 0.915 & 0.06459 \\
\hline 30 & 1,294 & 0.905 & 12.04 & 394 & 1,589 & 158 & 0.941 & 0.06468 \\
\hline 31 & 1,310 & 0.909 & 12.04 & 404 & 1,603 & 143 & 0.897 & 0.06469 \\
\hline 32 & 1,326 & 0.912 & 12.05 & 415 & 1,616 & 138 & 0.909 & 0.06475 \\
\hline 33 & 1,341 & 0.915 & 12.05 & 426 & 1,629 & 134 & 0.921 & 0.0648 \\
\hline 34 & 1,355 & 0.917 & 12.07 & 437 & 1,640 & 130 & 0.932 & 0.06489 \\
\hline 35 & 1,368 & 0.92 & 12.07 & 445 & 1,651 & 127 & 0.942 & 0.0649 \\
\hline 36 & 1,382 & 0.922 & 12.08 & 456 & 1,662 & 124 & 0.954 & 0.06499 \\
\hline 37 & 1,394 & 0.925 & 12.08 & 464 & 1,673 & 121 & 0.964 & 0.06499 \\
\hline 38 & 1,407 & 0.927 & 12.09 & 475 & 1,683 & 118 & 0.975 & 0.06506 \\
\hline 39 & 1,419 & 0.929 & 12.1 & 484 & 1,693 & 115 & 0.985 & 0.06511 \\
\hline 40 & 1,432 & 0.931 & 12.11 & 495 & 1,703 & 112 & 0.997 & 0.06517 \\
\hline 41 & 1,442 & 0.933 & 12.11 & 502 & 1,712 & 110 & 1.006 & 0.0652 \\
\hline 42 & 1,453 & 0.934 & 12.13 & 512 & 1,720 & 108 & 1.016 & 0.06529 \\
\hline 43 & 1,464 & 0.936 & 12.13 & 521 & 1,729 & 105 & 1.026 & 0.06532 \\
\hline 44 & 1,477 & 0.938 & 12.14 & 531 & 1,739 & 103 & 1.038 & 0.06538 \\
\hline 45 & 1,487 & 0.94 & 12.14 & 539 & 1,747 & 101 & 1.048 & 0.0654 \\
\hline & & & & & & & & \\
\hline
\end{tabular}

